\title{
Modeling Electromagnetic Form Factors of Light and Heavy Pseudoscalar Mesons
}

\author{
B. El-Bennich ${ }^{1,2}$, J. P. B. C. de Melo $^{3}$, B. Loiseau ${ }^{1}$, J.-P. Dedonder ${ }^{1}$, and T. Frederico ${ }^{4}$ \\ ${ }^{1}$ Laboratoire de Physique Nucléaire et de Hautes Énergies, Groupe Théorie, \\ CNRS, IN2P3 \& Universités Pierre et Marie Curie et Paris-Diderot, 75005 Paris, France \\ 2 Physics Division, Argonne National Laboratory, Argonne, IL 60439, USA \\ ${ }^{3}$ Laboratório de Física Teórica e Computação Científica, \\ Centro de Ciências Exatas e Tecnológicas, Universidade Cruzeiro do Sul, \\ 08060-700 and Instituto de Física Teorica, 01405-900, São Paulo, SP, Brazil and \\ ${ }^{4}$ Dep. de Física, Instituto Tecnológico de Aeronáutica, 12.228-900 São José dos Campos, SP, Brazil
} (Received on 12 May, 2008)

\begin{abstract}
The electromagnetic form factors of light and heavy pseudoscalar mesons are calculated within two covariant constituent-quark models, viz., a light-front and a dispersion relation approach. We investigate the details and physical origins of the model dependence of various hadronic observables: the weak decay constant, the charge radius and the elastic electromagnetic form factor.
\end{abstract}

Keywords: Light-front; Covariance; Constituent quark model; Electromagnetic current; Electromagnetic form factor

\section{INTRODUCTION}

The light-front quantum field theory is discussed by N. Bogoliubov et al. in Ref. [1] and an early application to study hadronic quark bound states may be found in Refs. [2, 3]. A self-consistent relativistic treatment of the quark spins can be performed within the light-front quark model, which allows for a calculation of the reference-frame independent partonic contribution to the form factor. The non-partonic contribution cannot be generally calculated, however it can be eliminated for space-like momentum transfers by an appropriate choice of reference frame. Therefore, the partonic contribution obtained in this specific reference frame yields the full form factor.

The light-meson sector, which includes the pion and kaon, allows us to test QCD hypotheses on the subatomic structure of hadrons at low and intermediate energies. Yet, many aspects of quantum field theory on the light-front and its application to bound-state systems give rise to various open questions; for example, the problems of regularization and renormalization on the light-front. For the pion and kaon, the light-front constituent quark model (LFCQM) has been studied $[4,5]$ to describe recent experimental data [6-9].

The description of these bound states using dispersion relations (DR) was first developed in [10]. It was applied to calculate light mesons form factors $[11,12]$ and used to elucidate long-distance effects $[13,14]$ in weak decays of heavy mesons. More recently the interplay between perturbative and nonperturbative regions in the pion electromagnetic form factor was discussed within the DR approach [15], where it was pointed out the dominance of the nonperturbative contribution up to rather high values of momentum transfers, going beyond a previous analysis performed up to intermediate values of momentum transfers within light-cone sum rule approach [16].

The DR approach is based on a consistent treatment of the two-particle singularities which arise in the triangle diagrams describing elastic as well as inelastic meson-transition amplitudes. In this dispersive approach, these amplitudes are given by relativistic spectral integrals over the mass variables in terms of Bethe-Salpeter amplitudes of the mesons and spectral densities of the corresponding triangle diagrams. Thus, elastic form factors are described by double spectral representations.

In this paper, we analyze the model dependence which, due to vertex functions as well as the choice of a constituent quark mass, usually arises in quark model calculations of elastic form factors. In the light sector, we compute the pion and kaon electric form factors for which experimental data is available; we then use our models to make predictions for the charmed sector. For simplicity we will assume point-like constituent quarks, as our model assumptions are made directly on the explicit form of wave functions and vertices. However, the constituent quark may have a structure that depends on the dynamical model of the bound state (see e.g. [17]).

\section{ELECTROMAGNETIC FORM FACTORS}

The space-like electromagnetic form factor of a pseudoscalar meson with mass $M$ is generally given by the covariant expression

$$
\left\langle P\left(p^{\prime}\right)\left|J_{\mu}^{\mathrm{em}}\right| P(p)\right\rangle=\left(p+p^{\prime}\right)_{\mu} F^{\mathrm{em}}\left(q^{2}\right),
$$

with $p^{2}=p^{\prime 2}=M^{2}$ and the four-momentum transfer $q=p^{\prime}$ $p, q^{2}<0$. The electromagnetic current is $J_{\mu}^{\mathrm{em}}=\bar{q}(0) \gamma_{\mu} q(0)$ where $q(0)$ denotes a current quark. Here, $F^{\mathrm{em}}\left(q^{2}\right)$ describes the virtual photon emission (absorption) amplitude by the composite state of a quark of charge $e_{1}$ and an antiquark of charge $e_{2}$. This form factor depends on the three independent Lorentz invariants $p^{2}, p^{2}$ and $q^{2}$. The constituent quark amplitude of the electromagnetic form factor is assumed to have the following structure

$$
\left\langle Q\left(p^{\prime}\right)\left|\bar{q}(0) \gamma_{\mu} q(0)\right| Q(p)\right\rangle=\bar{Q}\left(p^{\prime}\right) \gamma_{\mu} Q(p) \xi_{c}\left(q^{2}\right)
$$

where $Q(p)$ represents the constituent quark. The form factor is normalized such that $F^{\mathrm{em}}(0)=e_{1}+e_{2}$ and we neglect the anomalous magnetic moment of the constituent quark in Eq. (2). The function $\xi_{c}\left(q^{2}\right)$ describes a constituent quark 
transition form factor. Since the quark model is not formally derived from QCD, it is unknown. In the following we assume that $\xi_{c} \simeq e_{c}=e_{1}$ or $e_{2}$ owing to the fact that constituent quarks behave like bare Dirac particles [18].

\section{LIGHT-FRONT CONSTITUENT QUARK MODEL}

The electromagnetic form factor for pseudoscalar particles is calculated in the impulse approximation, using the Mandelstam formula

$$
\begin{gathered}
F^{\mathrm{em}}\left(q^{2}\right)=\frac{e_{1} N_{c}}{\left(p+p^{\prime}\right)^{\mu}} \int \frac{d^{4} k}{(2 \pi)^{4}} \operatorname{Tr}\left[\gamma^{5} S_{1}\left(k-p^{\prime}\right) \times\right. \\
\left.\gamma^{\mu} S_{1}(k-p) \gamma^{5} S_{2}(k)\right] \Lambda(k, p) \Lambda\left(k, p^{\prime}\right)+[1 \leftrightarrow 2],
\end{gathered}
$$

where $S_{i}(p)$ is the Feynman propagator of quark $i$ with constituent mass $m_{i}, N_{c}$ the number of colors, and $\Lambda(k, p)$ is our hadron-quark vertex function model. The bracket $[1 \leftrightarrow 2]$ is a shortcut for the subprocess of constituent quark 2 interacting with the photon.

The momentum component Bethe-Salpeter (BS) vertex model is chosen such that it regularizes the amplitude of the photo-absorption process and constructs a light-front valence wave function $[4,5,19]$. In the present study, we use two different models for the vertex functions taken from previous work, one of which has a non-symmetrical form under the exchange of the momentum of the quark and antiquark [4],

$$
\Lambda(k, p)=\frac{N}{(p-k)^{2}-m_{R}^{2}+i \varepsilon},
$$

while the other has a symmetric form [5],

$$
\Lambda(k, p)=\left[\frac{N}{k^{2}-m_{R}^{2}+i \varepsilon}+\frac{N}{(p-k)^{2}-m_{R}^{2}+i \varepsilon}\right] .
$$

The normalization constant $N$ is obtained from $F^{\mathrm{em}}(0)=e_{1}+$ $e_{2}$.

The light-front constituent quark model for hadrons used here is based on quantum field theory and our ansatz comes with the choice of the BS vertex and point-like quarks. It is worthwhile to mention that the present LFCQM reproduces in its full complexity a covariant calculation. Technically, the use of the Drell-Yan frame (longitudinal momentum transfer $q^{+}=q^{0}+q^{3}=0$ ) and the projection on to the light-front (performing the integration on the minus momentum component analytically in the Mandelstam formula) simplifies drastically the computation of the form factor. This approach has been applied to the pion [4, 5, 19], the kaon [20] as well as to the $\rho$ [21].

The use of light-front variables in the evaluation of the Mandelstam formula, with the corresponding projection on the light-front hypersurface through the $k^{-}=k^{0}-k^{3}$ integration, has its subtleties. Covariance of the starting expression in Eq. (3), which corresponds to a frame independent form factor, is an important property to be checked in the final results. In some cases, as for vector mesons in the present model, it is necessary to perform a careful analysis of the light-front calculation of the photo-absorption amplitude which accounts for pair terms or Z-diagrams that survive in the Drell-Yan frame (see, e.g., Ref. [22]). The Z-diagram vanishes in the calculation of the plus component of the electromagnetic current $\left(j^{+}\right)$ with $q^{+}=0$ for pseudoscalar mesons with $\gamma 5$ coupling and the momentum component of the BS vertices from Eqs. (4) and (5).

Therefore, we adopt the plus-component convention of the electromagnetic current in the Breit-frame with the DrellYan condition $\left(q^{+}=0\right)$ to compute the form factor of pseudoscalar mesons. The $k^{-}$integration is performed analytically by evaluating the residues in Eq. (3). In the case of the nonsymmetric vertex (4), the resulting expression for the electromagnetic form factor is:

$$
\begin{gathered}
F^{\mathrm{em}}\left(q^{2}\right)=e_{1} \frac{N^{2} N_{c}}{\left(p^{+}+p^{++}\right)} \int \frac{d^{2} k_{\perp} d k^{+}}{4 \pi^{3}} \frac{\left.\operatorname{Tr}_{1}^{+}[]\right|_{k^{-}=k_{2 \mathrm{on}}^{-}}}{k^{+}\left(p^{+}-k^{+}\right)^{2}} \\
\times \frac{\left.\left.\Lambda(k, p)\right|_{k^{-}=k_{\text {2on }}^{-}} \Lambda\left(k, p^{\prime}\right)\right|_{k^{-}=k_{2 \mathrm{on}}^{-}}}{\left(p^{-}-k_{2 \mathrm{on}}^{-}-(p-k)_{1 \mathrm{on}}^{-}\right)\left(p^{\prime-}-k_{2 \mathrm{on}}^{-}-\left(p^{\prime}-k\right)_{1 \mathrm{on}}^{-}\right)} \\
\quad+[1 \leftrightarrow 2],
\end{gathered}
$$

where the on-minus-shell momentum is $k_{1 \text { on }}^{-}=\left(k_{\perp}^{2}+m_{1}^{2}\right) / k^{+}$ $\left(k_{2 \text { on }}^{-}=\left(k_{\perp}^{2}+m_{2}^{2}\right) / k^{+}\right)$and the Dirac trace from Eq. (3) for $\mu=+($ good component of the current) is

$$
\begin{aligned}
\operatorname{Tr}_{1}^{+}[]= & -4\left[\left(p^{+}-k^{+}\right)\left(2 m_{1} m_{2}+\left(p+p^{\prime}\right) \cdot k-2 k^{2}\right)\right. \\
& \left.-k^{+}\left(\left(p^{\prime}-k\right) \cdot(p-k)-m_{1}^{2}\right)\right] .
\end{aligned}
$$

The electromagnetic form factor written for the nonsymmetric vertex (4) in terms of the Bjorken momentum fraction $x=k^{+} / p^{+}$is given by

$$
\begin{aligned}
& F^{\mathrm{em}}\left(q^{2}\right)=e_{1} \frac{N^{2} N_{c}}{8 \pi^{3}} \int \frac{d^{2} k_{\perp} d x}{x(1-x)^{4}} \\
& \quad \times \frac{\xi\left(x, \vec{k}_{\perp}, \vec{q}_{\perp}, m_{1}, m_{2}\right)}{\left(M^{2}-M_{0}^{\prime 2}\right)\left(M^{2}-M^{\prime 2}\left(m_{2}, m_{R}\right)\right)} \\
& \quad \times \frac{1}{\left(M^{2}-M_{0}^{2}\right)\left(M^{2}-M^{2}\left(m_{2}, m_{R}\right)\right)}+[1 \leftrightarrow 2],
\end{aligned}
$$

where $\xi\left(x, \vec{k}_{\perp}, \vec{q}_{\perp}, m_{1}, m_{2}\right)=\left.\left(p^{+}\right)^{-1} \operatorname{Tr}_{1}^{+}[]\right|_{k^{-}=k_{2 \text { on }}^{-}}, 0<x<1$ and the free two-quark mass is $M_{0}^{2}=M^{2}\left(m_{2}, m_{1}\right)$, with

$$
M^{2}\left(m_{i}, m_{R}\right)=\frac{k_{\perp}^{2}+m_{i}^{2}}{x}+\frac{(p-k)_{\perp}^{2}+m_{R}^{2}}{1-x}-p_{\perp}^{2} .
$$

Analogous expressions follow for $M_{0}^{\prime 2}$ and $M^{\prime 2}\left(m_{2}, m_{R}\right)$ with $p_{\perp}^{2}$ replaced by $p_{\perp}^{\prime 2}$. The calculation of the electromagnetic form factor with the symmetrical vertex (5) can be done following the steps presented above. For the interested reader, the derivation of the form factor for the pion in this model is performed in detail in Ref. [5].

The mass parameters in these models are limited by $m_{1}+$ $m_{2}>M, m_{1}+m_{R}>M$ and $m_{2}+m_{R}>M$ due to the unphysical continuum threshold as seen in the denominator of Eq. (8). Quark confinement does not allow for a scattering cut, but 


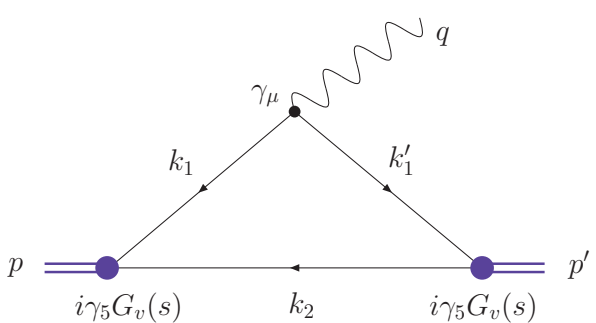

FIG. 1: The electromagnetic form factor in the impulse approximation is obtained from the triangle diagram. The vertices and momenta depicted in the diagram represent the momentum assignment in the DR approach.

since in the present model the meson is as a real bound state, i.e. the conditions $m_{1}+m_{2}>M$ and $m_{i}+m_{R}>M$ are satisfied, the cut is harmless. However, if the meson is a weakly bound composite particle, the form factor will be very sensitive to changes in the constituent quark mass. This is certainly not the case for pions and kaons, as they are Goldstone bosons and strongly bound, yet this sensitivity appears in the case of heavy pseudoscalars with small binding energies in nonconfining models (see, e.g., Ref. [23, 24]). In particular, our numerical results for $D^{+}$will exemplify the strong dependence of the heavy pseudoscalar electromagnetic form factor on the constituent masses, whereas this effect is minor for confining models like the DR approach.

\section{DISPERSION RELATION APPROACH}

The amplitude is obtained from the triangle diagram shown in Fig. 1 where the kinematical variables are displayed. The on-shell meson momenta are $p^{2}=M^{2}$ and $p^{2}=M^{2}$. The triangle diagram may be calculated in various ways - we choose to put the constituent quarks on-mass shell while keeping the external momenta off-shell with

$$
\tilde{p}^{2}=s, \quad \tilde{p}^{\prime 2}=s^{\prime}, \quad\left(\tilde{p}^{\prime}-\tilde{p}\right)^{2}=q^{2} .
$$

Note, however, that $\tilde{p}^{\prime}-\tilde{p}=\tilde{q} \neq q$.

In the DR approach, the electromagnetic form factors $F^{\mathrm{em}}\left(q^{2}\right)$ are expressed by a double spectral representation (see references [10-14]),

$$
\begin{aligned}
F^{\mathrm{em}}\left(q^{2}\right) & =e_{1} \int \frac{d s G_{v}(s)}{\pi\left(s-M^{2}\right)} \frac{d s^{\prime} G_{v}\left(s^{\prime}\right)}{\pi\left(s^{\prime}-M^{2}\right)} \\
& \times \Delta\left(s, s^{\prime}, q^{2} \mid m_{1}, m_{2}\right)+[1 \leftrightarrow 2] .
\end{aligned}
$$

We apply the Landau-Cutkosky rules to calculate the double spectral density $\Delta_{V}$ : we place all internal particles on their mass shell, $k_{1}^{2}=m_{1}^{2}, k_{1}^{\prime 2}=m_{1}^{2}, k_{2}^{2}=m_{2}^{2}$ but take the variables $p^{2}$ and $p^{\prime 2}$ off-shell. The double spectral density is then derived from calculation of the triangle diagram

$$
\begin{aligned}
2 \tilde{p}_{\mu}(q) \Delta\left(s, s^{\prime}, q^{2} \mid\right. & \left.m_{1}, m_{2}\right)=\frac{1}{8 \pi} \int d^{4} k_{1} d^{4} k_{1}^{\prime} d^{4} k_{2} \delta\left(k_{1}^{2}-m_{1}^{2}\right) \delta\left(k_{1}^{\prime 2}-m_{1}^{2}\right) \delta\left(k_{2}^{2}-m_{2}^{2}\right) \\
& \times \delta\left(\tilde{p}-k_{1}-k_{2}\right) \delta\left(\tilde{p}^{\prime}-k_{1}^{\prime}-k_{2}\right) \operatorname{Tr}\left[-\left(k_{1}^{\prime}+m_{1}\right) \gamma^{\mu}\left(k_{1}+m_{1}\right) i \gamma^{5}\left(m_{2}-k_{2}\right) i \gamma^{5}\right],
\end{aligned}
$$

with $\tilde{p}_{\mu}(q)=\tilde{p}_{\mu}-\frac{\tilde{p} \cdot q}{q^{2}} q_{\mu}$ which ensures the Ward identity and thus charge conservation $F^{\mathrm{em}}(0)=e_{1}+e_{2}$ with the proper vertex normalization.

The vertex of the pseudoscalar meson in the constituent quark picture has the structure

$$
\frac{\bar{Q}^{a}\left(k_{1}, m_{1}\right) i \gamma^{5} Q^{a}\left(-k_{2}, m_{2}\right)}{\sqrt{N_{c}}} G_{v}(s),
$$

where $Q^{a}\left(k_{1}, m_{1}\right)$ represents the spinor state of the constituent quark of color $a$ and $N_{c}$ the number of colors. The bound state vertex function may be related to the mesonic BS amplitude by

$$
\phi(s)=\frac{G_{v}(s)}{s-M^{2}} .
$$

For a confining potential, the pole at $s=M^{2}$ should appear in the physical region for $\phi(s)$ at $s=M^{2}>\left(m_{1}+m_{2}\right)^{2}$. This is, however, not the case; as well known from the behavior of the bound-state wave function in an harmonic oscillator potential, $\phi(s)$ is a smooth exponential function of $s \geq\left(m_{1}+m_{2}\right)^{2}$. This means that the would-be pole in $\phi(s)$ at $s=M^{2}$ is completely blurred out by the interaction and it is therefore more appropriate to analyze the meson form factors in terms of $\phi(s)$ rather than $G_{v}(s)$.

For a pseudoscalar meson in the dispersion approach of the constituent quark model, the BS amplitude $\phi(s)$ which accounts for soft constituent quark rescattering is given by [13]

$$
\phi(s)=\frac{\pi}{\sqrt{2}} \sqrt{\frac{s^{2}-\left(m_{1}^{2}-m_{2}^{2}\right)^{2}}{s-\left(m_{1}-m_{2}\right)^{2}}} \frac{w(k)}{s^{3 / 4}} .
$$

The dynamical factor multiplying $w(k)$ stems from the loop diagram associated with the meson-vertex normalization. The modulus of the center of mass momentum is

$$
k=\sqrt{\frac{\left(s+m_{1}^{2}-m_{2}^{2}\right)^{2}-4 s m_{1}^{2}}{4 s}} .
$$


It can be shown [13] that the vertex normalization of $G_{v}(s)=\phi(s)\left(s-M^{2}\right)$, which describes soft constituent rescattering, reduces to a simple normalization of the wave function

$$
\int_{0}^{\infty} w^{2}(k) k^{2} d k=1
$$

A heuristic choice must be made for $w(k)$. For phenomena that are predominantly governed by infrared mass scales, it is sensible in the case of heavy mesons to choose a BS amplitude parameterized by a function whose support is in the infrared; for instance functions of Gaussian form,

$$
w(k)=N \exp \left(-4 v k^{2} / \mu^{2}\right),
$$

where the reduced mass of the quark-antiquark pair is $\mu=$ $m_{1} m_{2} /\left(m_{1}+m_{2}\right)$ and $N$ a normalization constant. For light pseudoscalar mesons, dynamical chiral symmetry and the Ward-Takahashi identity are of great importance for their structure and properties. In non-perturbative approaches, using for example Schwinger-Dyson equations which incorporate these QCD features, appropriate parameterizations for the pion or kaon BS amplitudes are derived [25]. In this work, we employ power-law wave functions of the form

$$
w_{n}(k)=\frac{N}{\left(1+v(k / \mu)^{2}\right)^{n}}, \quad n \geq 2,
$$

inspired by the form suggested in Ref. [26] from the analysis of the ultraviolet physics of QCD.

The size parameter $v$ is to be determined from experimental and theoretical considerations. On the experimental side, on can either choose to constrain the vertex functions, or equivalently the BS amplitude, by the weak decay constant or by the electric charge radius. It is worthwhile to observe that both wave function models, Gaussian and power-law, do not present the singularity problem brought by the scattering cut due to changes the constituent quark masses, differently from the present LFCQM.

\section{NUMERICAL RESULTS}

The electromagnetic form factor of light and heavy pseudoscalar mesons are calculated with the two covariant constituent quark models. In the LFCQM, the quarks are in a bound state while in the DR model the wave functions corresponds to confined quarks with Gaussian and power-law forms. The shape of these functions is obtained from a fit to the experimental values of $f_{\pi}, f_{K}$ and $f_{D}$ or the charge radius (when available) of the regulator mass $m_{R}$ in the LFCQM and size parameter $v$ in the DR approach. The light constituent quark masses $m_{u}=m_{d}$ are allowed to take the values 0.22 and $0.25 \mathrm{GeV}$ (and $0.28 \mathrm{GeV}$ in case of the $D^{+}$). The strange and charm constituent masses are fixed to $m_{s}=0.508 \mathrm{GeV}$ and $m_{c}=1.623 \mathrm{GeV}$, respectively (see Ref. [27]).

The results for the pion electromagnetic form factor compared to experimental data [6-8] are shown in Fig. 2. The quark masses used in these calculations are $m_{u}=m_{d}=$ $0.22 \mathrm{GeV}$ in both the LFCQM and DR approaches. The

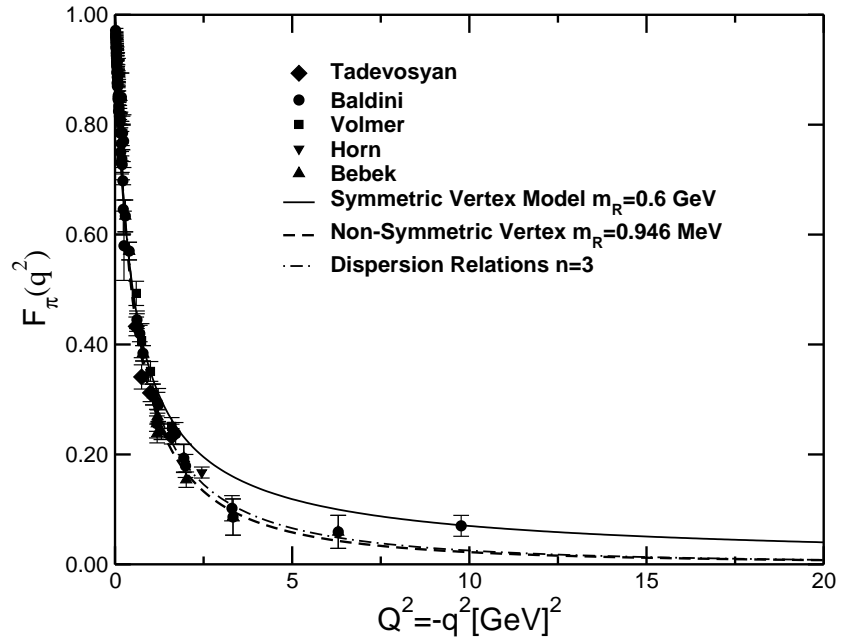

FIG. 2: The pion electormagnetic form factor in the LFCQM and DR approaches compared with experimental data from Ref. [6-9]. The model and respective parameters are explained in the legend.

pion charge radius is used to fit the parameters $m_{R}$ and $v$ (power-law wave function with $n=3$ ). The regulator mass is $m_{R}=0.946 \mathrm{GeV}$ [4] for the non-symmetric vertex and $0.546 \mathrm{GeV}$ [5] the symmetric vertex. In this case, we show results only for the power-law wave function with $n=3$ $(v=0.088)$ in the DR approach because the Gaussian vertex produces a form factor strongly damped with increasing $q^{2}$, which violates the asymptotic QCD result predicting a $q^{-2}$ falloff. The pion electromagnetic form factor for the powerlaw wave function and non-symmetric vertex are very similar, while the symmetrical model has a longer tail. These results indicate that the electromagnetic form factor of the pion as a strongly bound system does not particularly distinguish between models with a scattering cut. The high momentum experimental data appear to favor the more complex structure of the symmetrical vertex. It is expected that in this region the details of the regulator structure are crucial, and moreover a symmetric form of the vertex is natural.

We remind that the tail of the momentum component of the pion light-front wave function corresponding to the nonsymmetric [4] and to the symmetric vertex [5] both decrease as $\sim k_{\perp}^{-4}$, which can also be verified by inspecting Eq. (8). The power-law wave function model with $n=3$ also displays a tail that falls off with $\sim k_{\perp}^{-4}$. Thus, in the space-like region up to $10 \mathrm{GeV}^{2}$, we find that the pion electromagnetic form factor favors the power-law damping of the wave function with a $\sim k_{\perp}^{-4}$ tail.

The electromagnetic form factor of the $K^{+}$obtained in both models are compared with experimental data [28, 29] in Fig. 3. The quark masses used in these calculations are $m_{u}=m_{d}=0.22 \mathrm{GeV}$ and $m_{s}=0.508 \mathrm{GeV}$ in both the nonsymmetric vertex and DR approaches. In order to study the mass dependence of the form factor, we also give results for a calculation with $m_{u}=m_{d}=0.25 \mathrm{GeV}$ in LFCQM case. The regulator mass is fixed to the pion's value $m_{R}=0.946 \mathrm{GeV}$ for the non-symmetric vertex which corresponds to a reason- 


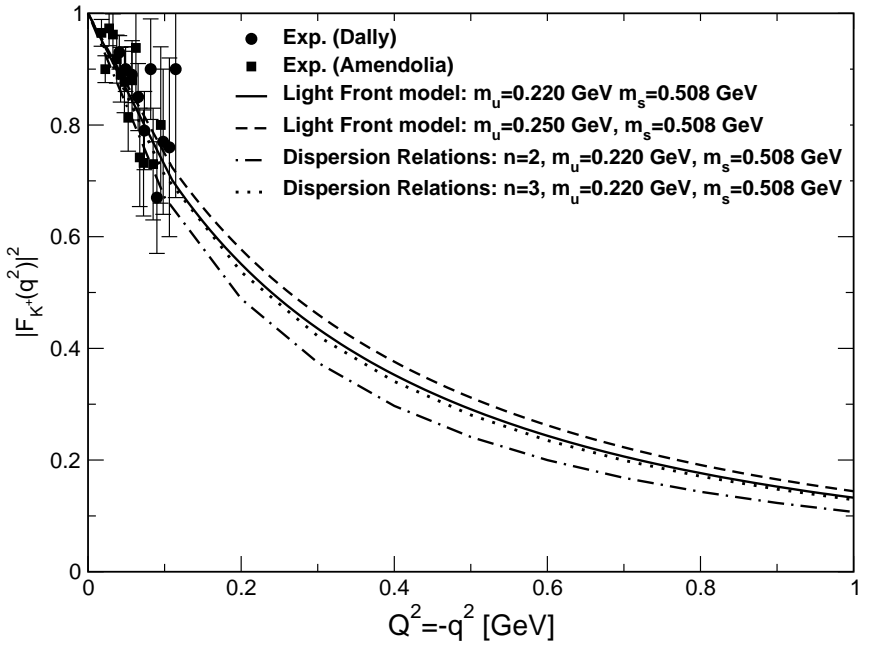

FIG. 3: The kaon electromagnetic form factor in the LFCQM and DR approaches compared with experimental data from Refs. [28, 29]. The models and respective parameters are explained in the legend

able kaon charge radius [20]. We observe in Fig. 3 that the change of $0.03 \mathrm{GeV}$ in the constituent quark mass in is not important as it is much smaller than the kaon binding energy of about $0.2 \mathrm{GeV}$. A similar finding can be reported for the DR approach, where the size parameter $\mu$ of the power-law wave function with $n=2$ is fitted $(v=0.061)$ to reproduce the kaon charge radius and is readjusted for $n=3(v=0.158)$. Again, we observe that the non-symmetric vertex as well as the power-law model essentially yield the same form factor once the radius is reproduced.

Our numerical results for $D^{+}$are shown in Fig. 4 for the DR approach and LFCQM. The size parameter of the Gaussian and power-law $(n=3)$ models are fitted to $f_{D}$ for $m_{d}=$ $0.25 \mathrm{GeV}$. Up to $10 \mathrm{GeV}^{2}$ there is no sizable difference between the two calculations. This is reasonable as $m_{c}$ sets one large scale whereas the momentum transfer goes only up to $3 \mathrm{GeV}$, which is not large enough to discriminate between the models. The regulator mass of $1.77 \mathrm{GeV}$ in the non-symmetric vertex model is chosen so as to give a sample of the LFCQM results. Here, we want to illustrate the constituent quark mass sensitivity when the symmetric and nonsymmetric vertices are used. We remind that the mass parameters in these models are limited by $m_{1}+m_{2}>M, m_{1}+m_{R}>M$ and $m_{2}+m_{R}>M$ due to the unphysical continuum threshold as evident from the denominator in Eq. (8). The model binding energy of $D^{+}$is only a few tenths of $\mathrm{MeV}$, as can be inferred from the $D^{+}$and quark masses. Since the $D^{+}$meson mass is fixed to the experimental value of $1.8694 \mathrm{GeV}$ and $m_{c}=1.623 \mathrm{GeV}$, the light quark mass $m_{d}$ must be larger than the value $0.22 \mathrm{GeV}$ we used for the pion and kaon. The increase of the light quark constituent mass from 0.25 to $0.28 \mathrm{GeV}$ corresponds to a large increase in binding and consequently to a decrease of the radius, as seen in Fig. 4.

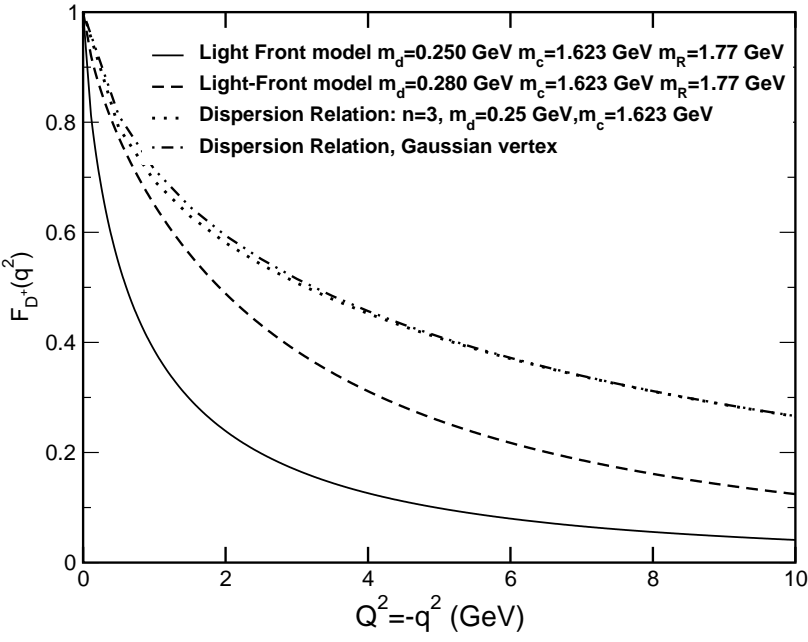

FIG. 4: Predictions for the $D^{+}$electromagnetic form factor in the LFCQM and DR approach.

\section{CONCLUSION}

We have concentrated on the model dependence of elastic electromagnetic form factors of light and heavy pseudoscalar mesons. Two different cases were studied by changing the form of the vertex or wave function and by varying the constituent quark masses. In the light sector, we computed the pion and kaon electric form factors for which experimental data is available; we then adjusted our models to make predictions for the charmed sector. Two ansatzes have been studied in the LFCQM which involve a symmetric and a nonsymmetric vertex form. In the DR approach, Gaussian and power-law wave functions were used in the calculations.

The form factors of the pion and kaon, both being strongly bound systems, are sensitive to the short-range part of the vertex functions. Therefore, the tail of the momentum component of the light-front wave functions for the non-symmetric [4] and symmetric vertex [5] as well as the power-law model ( $n=3$ ), all decreasing as $\sim k_{\perp}^{-4}$, is essential to reproduce the pion space-like data up to $10 \mathrm{GeV}^{2}$. Moreover, as a consequence of the strong binding of the quarks, changes in the constituent mass of about ten percent are not important for the elastic form factor of light mesons, provided the charge radius (or decay constant) is accordingly refitted. We have exemplified this in the kaon calculation. The light quark constituent masses are relevant for low momentum transfers of the order $m_{u, d, s}^{2}$ around $0.05-0.3 \mathrm{GeV}^{2}$, which is dominated by the charge radius and consequently compensated by fitting the size or regulator mass parameters. Therefore, in qualitative agreement with QCD scaling laws in the ultraviolet limit, a power-law form of the wave function and our choice of vertices are reasonable in reproducing the form factor data once the charge radius is fitted.

A recent calculation of the pion form factor within the dispersion relation approach including $O\left(\alpha_{S}\right)$ QCD perturbative 
contribution [15], showed in a model independent way that the nonperturbative part stays above $50 \%$ for $Q^{2} \leq 20 \mathrm{GeV}^{2}$. The nonperturbative part is compatible with a pion wave function close to the asymptotic one, with a momentum scale given by the effective continuum threshold that determines to a great extent their results for the form factor. The value of the threshold is extracted from the experimental pion decay constant. Similarly in our analysis the size or regulator mass parameters are fixed by the experimental values of the weak decay constants within models that have momentum tails decreasing with $\sim k_{\perp}^{-4}$, which does not leave not too much freedom for the pion form factor results below $20 \mathrm{GeV}^{2}$, as we have shown. In that respect our calculation and [15] show the dominance of the scale given by the pion decay constant that dials the vertex or wave function (decaying as a power law) in the pion form factor up to a fairly large value of momentum transfers.

The electromagnetic form factor of pseudoscalar mesons in the heavy-light sector is strongly sensitive to the infrared behavior of QCD, compared to the case of light mesons. The relevance of soft physics in this sector is supported by our calculations of the elastic $D$ meson form factor both within the dispersion relation and light-front approaches. Once the size parameter of the Gaussian and power-law wave functions are adjusted to reproduce the decay constant, the elastic form factor is insensitive to the vertex model in the space-like region up to $10 \mathrm{GeV}^{2}$. Therefore, the large-momentum tail of the wave function is not important for these results. We presume that the asymptotic behavior will be important only for $q^{2} \gg m_{c}^{2}$ beyond the results we presented. The importance of soft physics may also be appreciated in the covariant calculations of the form factor with the symmetric and nonsymmetric vertices in LFCQM. The corresponding BS amplitude models a lightly bound heavy-light system with respect to the light quark mass. The infrared dynamics appears to be important for the $D$ meson, as the sensitivity to changes of the constituent quark mass by about $10 \%$ suggests. This small modification produces a sensible change in the nearby unphysical continuum threshold, as the mass of the $D$ is about the sum of the constituent quark masses. Therefore, the condition $m_{1}+m_{2}>M$ is barely satisfied (see, e.g., the denominator of Eq. (8)).

In summary, our study of the model dependence of elastic form factors for pseudoscalar mesons in the light and heavy-light sectors suggests a separation of the ultraviolet and infrared physics. The ultraviolet properties of QCD dominate the form factor of light pseudoscalars, whereas infrared physics and details of quark confinement appear to be important for the space-like form factor of heavy mesons below $q^{2}=10 \mathrm{GeV}^{2}$. Hence, the electromagnetic form factors of heavy-light systems, such as the $D$ and $B$ mesons, provide a valuable tool in the effort to investigate the nonperturbative physics of confinement, in contrast with light pseudoscalar form factors insensitive to the details of infrared physics even at low momentum transfers once the weak decay constants are fixed to the experimental values.

\section{Acknowledgments}

J. P. B. C. M. and T. F. thank the LPNHE for the kind hospitality during their visits when part of this work was developed. B. E. is grateful to Lauro Tomio for a pleasant stay at the Instituto de Física Teórica and to the Universidade Cruzeiro do Sul for the welcoming atmosphere; he also enjoyed valuable discussions with C. D. Roberts. This work was supported by the Department of Energy, Office of Nuclear Physics, contract no. DE-AC02-06CH11357. We acknowledge bilateral funding from the Centre National de la Recherche Scientifique (CNRS) and Fundação de Amparo à Pesquisa do Estado de São Paulo FAPESP) under grant no. 06/50343-8 as well as partial support from the Conselho Nacional de Desenvolvimento Científico e Tecnológico (CNPq).
[1] N. N. Bogoliubov, V. A. Matveev, and A. N. Tavkhlidze, in "Gravitation and Elementary Particle Physics", ed. by A. A. Logunov (Mir, Moscow, 1983), p. 220.

[2] M. V. Terentev, Sov. J. Nucl. Phys. 24, 106 (1976) 106; L. A. Kondratyuk and M. V. Terentev, Sov. J. Nucl. Phys. 31, 561 (1976).

[3] V. B. Berestesky and M. V. Terentev, Sov. J. Nucl. Phys. 24, 547 (1976); Sov. J. Nucl. Phys. 25, 347 (1977).

[4] J. P .B. C de Melo, H. W. Naus, and T. Frederico, Phys. Rev. C 59, 2278 (1999).

[5] J. P. B. C de Melo, T. Frederico, E. Pace, and G. Salmè, Nucl. Phys. A 707, 399 (2002).

[6] R. Baldini et al., Eur. Phys. J. C 11709 (1999); Nucl. Phys. A 666\& 667, 3 (2000)

[7] The Jefferson Lab F(pi) Collaboration (J. Volmer et al.), Phys. Rev. Lett. 86, 1713 (2001).

[8] The Jefferson Lab F(pi) Collaboration (T. Horn et al.), Phys. Rev. Lett. 97, 192001 (2006).

[9] The Jefferson Lab F(pi) Collaboration (V. Tadevosyan et al.), Phys. Rev. C 75, 055205 (2007).
[10] V. V. Anisovich, M. N. Kobrinsky, D. Melikhov, and A. Sarantsev, Nucl. Phys. A544, 747 (1992).

[11] V. V. Anisovich, D. Melikhov, and V. Nikonov, Phys. Rev. D52, 5295 (1995).

[12] V. V. Anisovich, D. Melikhov, and V. Nikonov, Phys. Rev. D 55, 2918 (1997).

[13] D. Melikhov, Phys. Rev. D 53, 2460 (1996).

[14] D. Melikhov, Eur. Phys. J. C 4, 2 (2002).

[15] V. Braguta, W. Lucha, and D. Melikhov, Phys. Lett. B 661, 354 (2008).

[16] V. M. Braun, A. Khodjamirian, and M. Maul, Phys. Rev. D 61, 073004 (2000).

[17] W. Lucha, D. Melikhov, and S. Simula, Phys. Rev. D 74, 054004 (2006)

[18] S. Weinberg, Phys. Rev. Lett. 65, 1181 (1990).

[19] B. L. G. Bakker, H. M. Choi, and C. R. Ji, Phys. Rev. D 63, 074014 (2001)

[20] F. P. Pereira, J. P. B. C. de Melo, T. Frederico, and Lauro Tomio, Nucl. Phys. A 790, 610c (2007); Phys. Part. Nucl. 36, 217 (2005). 
[21] J. P. B. C. de Melo, T. Frederico, Phys. Rev. C 55, 2043 (1997).

[22] J. P. B. C. de Melo, J. H. O. Sales, T. Frederico, and P. U. Sauer, Nucl. Phys. A 631, 574c (1998).

[23] T. Frederico, H.-C. Pauli, Phys. Rev. D 64, 054007 (2001).

[24] L. A. M. Salcedo, J. P. B. C. de Melo, D. Hadjmichef, and T. Frederico, Braz. J. Phys. 34, 297 (2004); Eur. Phys. J. A 27, 213 (2006).

[25] P. Maris, C. D. Roberts, and P. C. Tandy, Phys. Lett. B 420, 267
(1998).

[26] F. Schlumpf, Phy. Rev. D 50, 6895 (1994).

[27] E. F. Suisso, J. P. B. C. de Melo, and T. Frederico, Phys. Rev. D 65, 094009(2002).

[28] E. B. Dally et al., Phys. Rev. Lett. 45, 232 (1980).

[29] S. R. Amendolia et al., Phys. Lett. 178, 435 (1986). 